\title{
A!
}

This is an electronic reprint of the original article.

This reprint may differ from the original in pagination and typographic detail.

Hekking, Frank; Pekola, Jukka

\section{Finite frequency quantum noise in an interacting mesoscopic conductor}

Published in:

Physical Review Letters

DOI:

10.1103/PhysRevLett.96.056603

Published: 09/02/2006

Document Version

Publisher's PDF, also known as Version of record

Please cite the original version:

Hekking, F., \& Pekola, J. (2006). Finite frequency quantum noise in an interacting mesoscopic conductor.

Physical Review Letters, 96(5), 1-4. [056603]. https://doi.org/10.1103/PhysRevLett.96.056603

This material is protected by copyright and other intellectual property rights, and duplication or sale of all or part of any of the repository collections is not permitted, except that material may be duplicated by you for your research use or educational purposes in electronic or print form. You must obtain permission for any other use. Electronic or print copies may not be offered, whether for sale or otherwise to anyone who is not an authorised user. 


\title{
Finite Frequency Quantum Noise in an Interacting Mesoscopic Conductor
}

\author{
F. W. J. Hekking ${ }^{1,2}$ and J.P. Pekola ${ }^{2}$ \\ ${ }^{1}$ Laboratoire de Physique et Modélisation des Milieux Condensés, CNRS \& Université Joseph Fourier, \\ Boîte Postale 166, 38042 Grenoble-cedex 9, France \\ ${ }^{2}$ Low Temperature Laboratory, Helsinki University of Technology, Post Office Box 3500, 02015 HUT, Finland
}

(Received 19 August 2005; published 9 February 2006)

\begin{abstract}
We present a quantum calculation based on scattering theory of the frequency-dependent noise of current in an interacting chaotic cavity. We include interactions of the electron system via long range Coulomb forces between the conductor and a gate with capacitance $C$. We obtain explicit results exhibiting the two time scales of the problem, the cavity's dwell time $\tau_{D}$ and the $R C$ time $\tau_{C}$ of the cavity in relation to the gate. The noise shows peculiarities at frequencies of the order and exceeding the inverse charge relaxation time $\tau^{-1}=\tau_{D}^{-1}+\tau_{C}^{-1}$.
\end{abstract}

DOI: 10.1103/PhysRevLett.96.056603

Electrical noise in mesoscopic conductors is known to yield a wealth of information on microscopic mechanisms of transport [1]. Recently, the focus in theory and experiment has shifted from studies of noise spectra to those of general current statistics including all the moments of current [2]. Meanwhile, though, important properties of the standard second moment, i.e., the part of statistics that is normally associated with noise, are still to be uncovered in several important aspects of direct relevance to experimental investigations. An example is finite-frequency noise of a mesoscopic conductor. The interest of studying it is twofold. First, one expects that the noise will probe the intrinsic dynamics of the conductor at the Thouless energy (inverse dwell time $\tau_{D}$ ). Second, at finite frequency, current is no longer spatially homogeneous, and charge piles up in the conductor. Coulomb interaction screens this pileup of charge, at a characteristic charge relaxation frequency which may well be different from $1 / \tau_{D}$. Therefore, any theoretical treatment of finite-frequency noise should take interaction effects into account [1]. Calculation of the noise spectrum of an arbitrary, interacting mesoscopic conductor constitutes a formidable task, which has so far been accomplished in specific cases only, like mesoscopic capacitors [1], Coulomb blockade systems [3] and Luttinger liquids [4]. Finite-frequency noise spectra are of experimental relevance, as they can be measured with recently developed quantum detectors [5] that distinguish finite positive and negative frequencies by separately probing emission and absorption.

In this Letter we present a self-consistent theory of high frequency quantum noise of a chaotic cavity. We calculate emission and absorption spectra with the help of scattering theory, including interactions to ensure current conservation. Besides providing timely guidelines for experiments on noise spectroscopy, our theory paves the way towards a consistent calculation of the finite-frequency higher moments of current, in particular, the presently actively investigated third cumulant [6]. We obtain the noise spectrum of a chaotic quantum cavity capacitively connected to a screening gate; see Fig. 1. Specifically we show that,
PACS numbers: 72.70.+m, 05.45.Mt, 73.23.-b

under realistic conditions, the noise spectra show a distinct enhancement at the inverse charge relaxation time $\tau^{-1}=$ $\tau_{D}^{-1}+\tau_{C}^{-1}$. The dwell time is $\tau_{D}=h N_{F} / N$, and the $R C$ time $\tau_{C}=h C /\left(N e^{2}\right)$, where $N_{F}$ is the density of states of the cavity at the Fermi level, $N=N_{L}+N_{R}$ is the sum of the total number of propagating electronic modes of the left and right lead, respectively, and $C$ is the geometric capacitance. Similar peculiarities may well occur in the spectra of other mesoscopic conductors at frequencies of the order of their inverse charge relaxation times.

In the scattering theory of quantum transport the current operator is given by [7]

$$
\begin{aligned}
\hat{I}_{\alpha}(t)= & \frac{e}{2 \pi \hbar} \sum_{n} \int d E d E^{\prime} e^{i\left(E-E^{\prime}\right) t / \hbar}\left[\hat{a}_{\alpha n}^{\dagger}(E) \hat{a}_{\alpha n}\left(E^{\prime}\right)\right. \\
& \left.-\hat{b}_{\alpha n}^{\dagger}(E) \hat{b}_{\alpha n}\left(E^{\prime}\right)\right] .
\end{aligned}
$$

The operator $\hat{a}_{\alpha n}^{\dagger}(E)(\hat{a})$ creates (annihilates) electrons incident upon the scatterer with energy $E$ in propagating mode $n$ in lead $\alpha$. Similarly, $\hat{b}^{\dagger}, \hat{b}$ denote electrons in the outgoing states. For the two-terminal setup depicted in Fig. 1(a), $\alpha$ takes values $L$ and $R$ for the left and right leads, respectively. The operators $\hat{a}$ and $\hat{b}$ are related by the scattering matrix $s, \hat{b}_{\alpha n}(E)=s_{\alpha \gamma ; n m}(E) \hat{a}_{\gamma m}(E)$ [8]. The matrix $s$ is unitary and has dimensions $N \times N$. Using $s$, we eliminate the $\hat{b}$ operators from Eq. (1). Defining the current matrix

$$
A_{\alpha \gamma ; m n}^{(0)}\left(L, E, E^{\prime}\right)=\delta_{m n} \delta_{L \alpha} \delta_{L \gamma}-s_{L \alpha ; m k}^{\dagger}(E) s_{L \gamma ; k n}\left(E^{\prime}\right),
$$

we write current in the left lead as
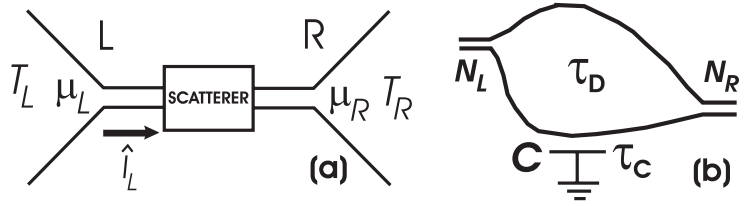

FIG. 1. (a) Two-terminal scattering problem. (b) Chaotic quantum cavity; the capacitor $C$ accounts for the interactions. 


$$
\begin{aligned}
\hat{I}_{L}(t)= & \frac{e}{2 \pi \hbar} \int d E d E^{\prime} e^{i\left(E-E^{\prime}\right) t / \hbar} \hat{a}_{\alpha m}^{\dagger}(E) \\
& \times A_{\alpha \gamma ; m n}^{(0)}\left(L, E, E^{\prime}\right) \hat{a}_{\gamma n}\left(E^{\prime}\right) .
\end{aligned}
$$

The superscript (0) indicates that we deal with noninteracting electrons.

The current-current correlation function in the left lead can now be written as $\left\langle\delta \hat{I}_{L}(t) \delta \hat{I}_{L}(0)\right\rangle=\int \frac{d \omega}{2 \pi} e^{-i \omega t} S_{L L}(\omega)$, where we denote $\delta \hat{I}_{L}(t) \equiv \hat{I}_{L}(t)-\left\langle\hat{I}_{L}(t)\right\rangle$. Using Eqs. (2) and (3), and performing thermal averaging of products of operators $\hat{a}$ and $\hat{a}^{\dagger}$ for equilibrium reservoirs, we obtain the noise spectrum in the absence of interactions [1]

$$
\begin{aligned}
S_{L L}^{(0)}(\omega)= & \frac{e^{2}}{h} \int d E \operatorname{Tr}\left[A_{\alpha \gamma}^{(0)}(L, E, E+\hbar \omega)\right. \\
& \left.\times A_{\gamma \alpha}^{(0)}(L, E+\hbar \omega, E)\right]\left\{f_{\alpha}(E)\left[1-f_{\gamma}(E+\hbar \omega)\right]\right. \\
& \left.+\left[1-f_{\alpha}(E)\right] f_{\gamma}(E+\hbar \omega)\right\} .
\end{aligned}
$$

Here $f_{\alpha}(E)$ is the Fermi function in lead $\alpha$, kept at tem- perature $T_{\alpha}$ and chemical potential $\mu_{\alpha}$ [see Fig. 1(a)]; the notation $A_{\alpha \gamma}^{(0)}\left(L, E, E^{\prime}\right)$ indicates the $N_{\alpha} \times N_{\gamma}$ block of the matrix $A^{(0)}\left(L, E, E^{\prime}\right) ; \operatorname{Tr}\left[C_{\alpha \alpha}\right]$ is the trace of the square matrix $C_{\alpha \alpha}$ which implies summing over the $N_{\alpha}$ propagating modes of lead $\alpha$. For energy-independent scattering, the integration over $E$ can be straightforwardly performed and Eq. (4) reduces to the result found in Ref. [9], valid for a tunnel barrier or a quantum point contact. However, quite generally the current matrix $A$ contains correlations at different energies separated by $\hbar \omega$ [10]. Here we consider the effect of these correlations on the finite-frequency noise of a chaotic quantum cavity, which can be treated in the framework of random matrix theory. Specifically, we make use of Ref. [11] to obtain the ensemble averaged, energydependent quantity $\left\langle\operatorname{Tr}\left[A_{\alpha \gamma}^{(0)}(L, E, E+\hbar \omega) A_{\gamma \alpha}^{(0)}(L, E+\right.\right.$ $\hbar \omega, E)]\rangle$ (no sum over $\alpha$ and $\gamma$ ). In view of Eq. (2), this amounts to the calculation of the average trace of the product of two scattering matrices, which we achieve with the help of Ref. [11], $\left\langle\operatorname{Tr}\left[s_{\alpha \gamma}^{\dagger}\left(E_{1}\right) s_{\alpha \gamma}\left(E_{2}\right)\right]\right\rangle \equiv$ $\left(N_{\alpha} N_{\gamma} / N\right) g\left(E_{2}-E_{1}\right)$, where $g(E)=\left[1-i E \tau_{D} / \hbar\right]^{-1}$. Similarly, we need

$$
\begin{aligned}
\left\langle\operatorname{Tr}\left[s_{\gamma \alpha}^{\dagger}\left(E_{1}\right) s_{\gamma \delta}\left(E_{2}\right) s_{\varepsilon \delta}^{\dagger}\left(E_{3}\right) s_{\varepsilon \alpha}\left(E_{4}\right)\right]\right\rangle= & \frac{N_{\alpha} N_{\gamma}}{N^{2}}\left[\delta_{\alpha \delta} N_{\varepsilon} g\left(E_{2}-E_{1}\right) g\left(E_{4}-E_{3}\right)+\delta_{\gamma \varepsilon} N_{\delta} g\left(E_{4}-E_{1}\right) g\left(E_{2}-E_{3}\right)\right. \\
& \left.-\frac{N_{\delta} N_{\varepsilon}}{N} \frac{g\left(E_{2}-E_{1}\right) g\left(E_{4}-E_{3}\right) g\left(E_{4}-E_{1}\right) g\left(E_{2}-E_{3}\right)}{g\left(E_{2}+E_{4}-E_{1}-E_{3}\right)}\right] .
\end{aligned}
$$

These results depend on the cavity's dwell time $\tau_{D}$ as well as on the number of modes $N_{L, R}$ in the leads. Certain inequalities apply to these quantities. First of all, we assume $\tau_{D}$ to be much longer than the Ehrenfest time that characterizes the spreading of a wave packet in the cavity [12]. This ensures that the electrons spend enough time in the cavity for quantum chaos to fully develop and random matrix theory to apply. Hence our results are universal in the sense that they do not depend on details of the cavity. Second, the results of Ref. [11] were obtained for a large scattering matrix, i.e., in the limit $N \gg 1$. We ignore corrections of relative order $\mathcal{O}(1 / N)$, i.e., weak localization is not taken into account. Finally, we also ignore renormalization of transport by interactions [13], assuming that both leads are wellconnected to the cavity with $N_{L}, N_{R} \gg 1$. However, the openings to the leads are much smaller than the total circumference of the cavity in order for universality to hold.

Performing the ensemble average of (4), we find the frequency-dependent noise of a noninteracting chaotic cavity at finite temperature $\beta=1 / k_{B} T$ and applied bias $V$,

$$
S_{L L}^{(0)}(\omega)=\frac{e^{2}}{h} \frac{N_{L} N_{R}}{N} \frac{1+\omega^{2} \tau_{D}^{2} N / N_{R}}{1+\omega^{2} \tau_{D}^{2}}\left\{\left[1-\frac{N_{L} N_{R}}{N^{2}}\right] \frac{2 \hbar \omega}{1-e^{-\beta \hbar \omega}}+\frac{N_{L} N_{R}}{N^{2}}\left[\frac{\hbar \omega+e V}{1-e^{-\beta(\hbar \omega+e V)}}+\frac{\hbar \omega-e V}{1-e^{-\beta(\hbar \omega-e V)}}\right]\right\} .
$$

We consider noise in the left lead; hence the dependence on $N_{L}, N_{R}$ is nonsymmetric. In the limit $\omega \rightarrow 0$, Eq. (5) reduces to the known result for zero-frequency noise of a chaotic cavity [14] which has been studied experimentally [15]. We will discuss the frequency-dependent spectral function below, after interactions have been included.

In order to treat the case of an interacting chaotic cavity we follow the general framework of Ref. [16]. The central idea is that fluctuations of particle current, as given by Eq. (1), lead to a pileup of charge in the cavity that is subsequently compensated by displacement currents induced at the contacts and the gate. The total current at a given contact can be written as the sum of particle current and displacement current at that contact. To determine the displacement current, the uniform self-consistent potential of the cavity must be found [17], a problem that can be solved explicitly within the random phase approximation $[16,18]$. The resulting total current operator (sum of particle and displacement current) for the left lead is still given by Eq. (3), however the current matrix $A^{(0)}$ is replaced by an effective current matrix $A=A^{(0)}+\Delta A$, where $\Delta A$ accounts for the displacement contribution. This guarantees current conservation at finite frequency: the sum of all currents at the contacts of the sample together with the displacement current at the gate is zero. The explicit form of $\Delta A$ reads [16] 


$$
\Delta A_{\gamma \delta}(\alpha, E, E+\hbar \omega)=i 2 \pi \hbar \omega G_{\alpha}(\omega) \mathcal{N}_{\gamma \delta}(E, E+\hbar \omega),
$$

where

$$
\mathcal{N}_{\gamma \delta}\left(E, E^{\prime}\right)=\frac{i}{2 \pi} \frac{s_{\alpha \gamma}^{\dagger}(E)\left[s_{\alpha \delta}(E)-s_{\alpha \delta}\left(E^{\prime}\right)\right]}{E^{\prime}-E}
$$

is the density of states matrix and $G_{\alpha}(\omega)$ a frequencydependent response function, proportional to the emittance [19] of the cavity. In the linear response regime,

$$
G_{\alpha}(\omega)=\frac{N_{\alpha}}{N} \frac{e^{2} N_{F}}{C+e^{2} N_{F}} \frac{1}{1-i \omega \tau},
$$

with a frequency dependence governed by the charge relaxation time $\tau$.

Turning to the noise, we replace $A^{(0)}$ by $A$ in Eq. (4) and find the additional contributions to noise related to the interaction correction $\Delta A$. Performing the ensemble averaging of these terms $\left(\left\langle A^{(0)} \Delta A\right\rangle,\left\langle\Delta A A^{(0)}\right\rangle\right.$, and $\left.\langle\Delta A \Delta A\rangle\right)$, we obtain the full noise spectral function: it is given by Eq. (5), upon replacing the dwell time $\tau_{D}$ by the charge relaxation time $\tau$. This is our central result, which we will discuss in several relevant limits.

If $V \rightarrow 0$, we find the nonsymmetrized noise spectrum,

$$
S_{L L}(\omega)=\frac{e^{2}}{h} \frac{N_{L} N_{R}}{N} \frac{2 \hbar \omega}{1-e^{-\beta \hbar \omega}} \frac{1+\omega^{2} \tau^{2} N / N_{R}}{1+\omega^{2} \tau^{2}} .
$$

The symmetrized equilibrium spectrum $\left[S_{L L}(\omega)+\right.$ $\left.S_{L L}(-\omega)\right] / 2$ is plotted in Fig. 2(a). According to the fluctuation dissipation theorem, this is proportional to the real part of the ac conductance $G_{L L}(\omega)=I_{L}(\omega) / V_{L}(\omega)$ of the cavity. We obtain

$$
\operatorname{Re}\left[G_{L L}(\omega)\right]=\frac{e^{2}}{h} \frac{N_{L} N_{R}}{N} \frac{1+\omega^{2} \tau^{2} N / N_{R}}{1+\omega^{2} \tau^{2}},
$$

in agreement with a direct calculation [20]. At equilibrium, the cavity corresponds to the effective circuit depicted in the inset of Fig. 2(a). Here, $G_{L}$ and $G_{R}$ are the quantum conductances of the left and right lead, respectively, whereas $C_{\mu}$ is the series capacitance of the true geometric capacitance $C$ and the quantum capacitance $e^{2} N_{F}$ of the cavity. At low frequencies, the effect of this capacitance can be ignored, and the noise is that of the series conductance $G=G_{L} G_{R} /\left(G_{L}+G_{R}\right)=\left(N_{L} N_{R} / N\right) e^{2} / h$. At high frequency, $C_{\mu}$ opens a new current path thereby increasing the ac conductance and hence the noise. The corresponding frequency scale is set by $\tau^{-1}$, which is the inverse $R C$ time of the two resistances $G_{L}^{-1}$ and $G_{R}^{-1}$ in parallel, in combination with the capacitance $C_{\mu}$. Quite generally, the noninteracting case corresponds to the limit of large geometric capacitance, such that the quantum capacitance-and hence the dwell time $\tau_{D}$-determines the dynamics. In this limit the noise spectra are obtained from Eq. (5). The result (9) for nonsymmetrized noise is plotted in Fig. 2(b). There is a pronounced difference between noise at negative

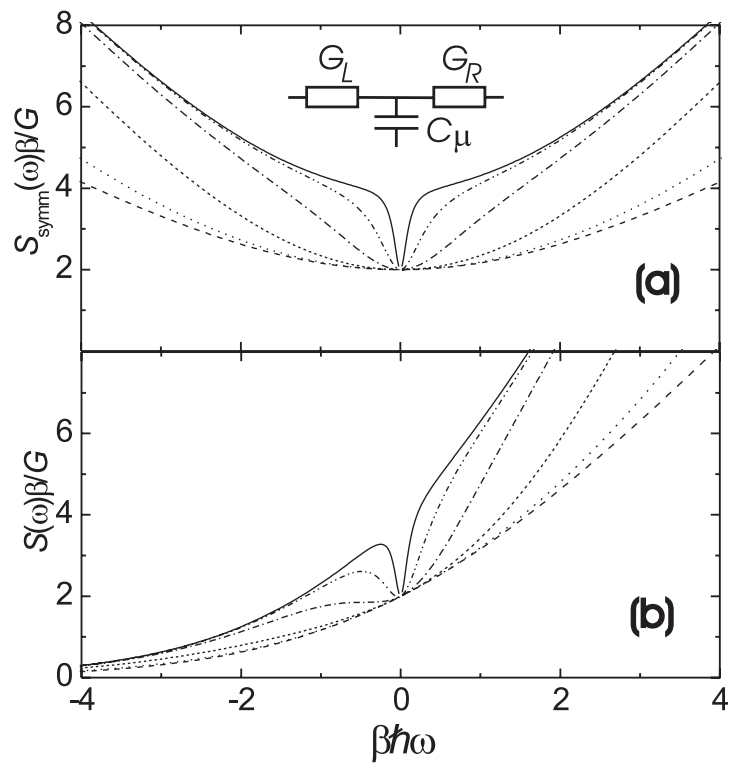

FIG. 2. (a) Frequency-dependent symmetrized noise $S_{\text {symm }}(\omega)$ in units $G / \beta$ of an interacting chaotic cavity with $N_{L} / N=0.5$ and charge relaxation times (from top) $\tau /(\hbar \beta)=10,3,1,0.3,0.1$; bottom curve corresponds to energy-independent scattering $\tau=$ 0. Inset: corresponding circuit model. (b) Frequency-dependent unsymmetrized quantum noise; parameters as in (a).

and positive frequencies, corresponding, respectively, to absorption and emission. For negative frequencies a peculiarity develops at $\omega \sim-\tau^{-1}$ as a manifestation of enhanced absorption due to charged fluctuations.

If $e V \gg k_{B} T, \hbar \omega$ we have the nonequilibrium noise

$$
S_{L L}(\omega)=\frac{N_{L} N_{R}}{N^{2}} e\langle I\rangle \frac{1+\omega^{2} \tau^{2} N / N_{R}}{1+\omega^{2} \tau^{2}},
$$

with $\langle I\rangle=G V$ the average current through the cavity. The Fano factor $F(\omega) \equiv S_{L L}(\omega) /(e\langle I\rangle)$ is plotted in Fig. 3(a). At zero frequency this gives the result $S_{L L}(0)=F(0) e\langle I\rangle$, with the noninteracting shot-noise reduction factor $F(0)=$ $N_{L} N_{R} / N^{2}$ [14]; the interaction correction is small in the parameter $1 / N$ [13]. At frequencies exceeding $1 / \tau$, the Fano factor increases by $N / N_{R}$. Let us also consider the time-dependent second cumulant of charge transport [21] for the left lead, given by

$$
\left\langle\left\langle q^{2}\right\rangle\right\rangle(t)=2 \int \frac{d \omega}{2 \pi} S_{L L}(\omega) \frac{1-\cos (\omega t)}{\omega^{2}} .
$$

Inserting (10) into (11) we have

$$
\left\langle\left\langle q^{2}\right\rangle\right\rangle(t)=\frac{N_{L} N_{R}}{N^{2}} e\langle I\rangle t\left[1+\frac{N_{L}}{N_{R}} \frac{\tau}{t}\left(1-e^{-t / \tau}\right)\right] .
$$

The time dependence of $\left\langle\left\langle q^{2}\right\rangle\right\rangle /\left(F_{0} e\langle I\rangle \tau\right)$ is plotted in Fig. 3(b). The universal long time limit is reached only after a time $\tau$; the ratio of the slopes at early and late times is determined by the asymmetry given by $N / N_{R}$. 

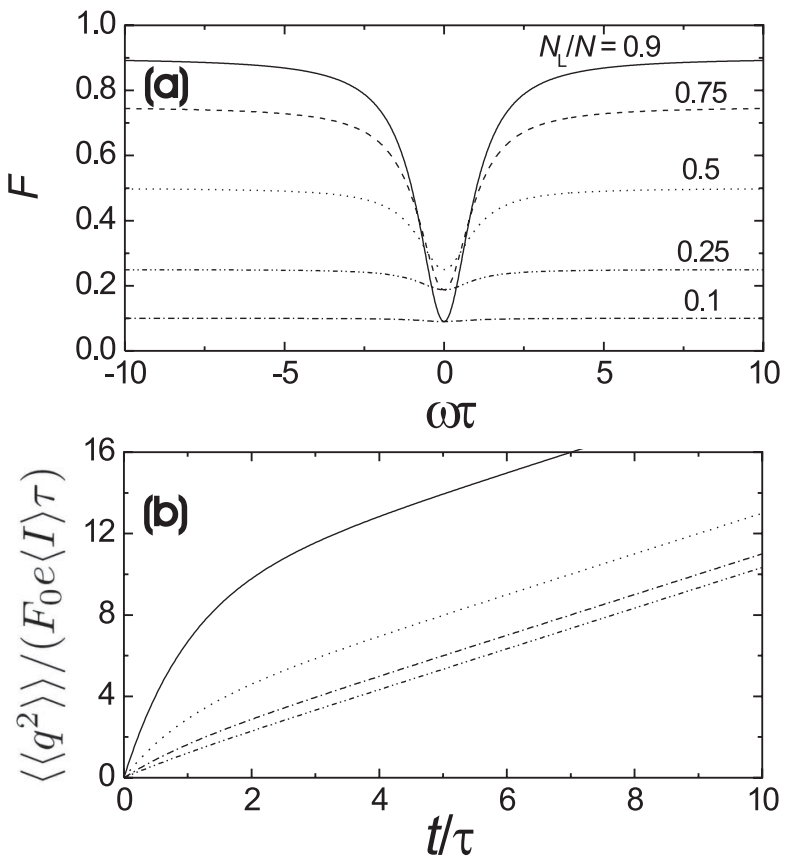

FIG. 3. (a) Frequency dependence of the Fano factor at a few values of $N_{L} / N$. (b) The second cumulant for a cavity with $N_{L} / N=0.9,0.75,0.5$, and 0.3 from top to bottom.

Finally, we address the feasibility of a measurement of the above found noise spectra. For the cavities studied in the experiment [15], the various conditions for our calculations to be valid appear to be met. The number of modes in the leads is relatively large, $N \sim 5-10$. The Ehrenfest time is of the order of $300 \mathrm{ps}$ and the dwell time $\tau_{D}$ in the nanosecond range. Given the size of a typical cavity, we estimate $\tau_{C}$ up to $1 \mathrm{~ns}$, thereby giving a charge relaxation time $\tau$ of the same order, still larger than the Ehrenfest time. The condition $\omega \tau \sim 1$ can then be reached well within the chaotic regime, using a Josephson junction in the quantum limit as a detector, where the level spacing (plasma frequency) is $\sim 10-100 \mathrm{GHz}$. Our results may be helpful for future experiments on other mesoscopic conductors also, such as a diffusive metallic wire capacitively coupled to a nearby ground plane [22].

We thank T. Heikkilä, F. Pistolesi, R. Whitney, and especially P. Brouwer, M. Büttiker, H. Schomerus, and D. Prober for useful discussions and comments. Financial support from Academy of Finland and from Institut Universitaire de France is gratefully acknowledged.

[1] Ya. M. Blanter and M. Büttiker, Phys. Rep. 336, 1 (2000).

[2] Quantum Noise in Mesoscopic Physics, edited by Yu. V. Nazarov (Kluwer, Dordrecht, 2003).
[3] G. Johansson, A. Käck, and G. Wendin, Phys. Rev. Lett. 88, 046802 (2002); A. Käck, G. Wendin, and G. Johansson, Phys. Rev. B 67, 035301 (2003); A. V. Galaktionov, D.S. Golubev, and A.D. Zaikin, ibid. 68, 085317 (2003).

[4] B. Trauzettel et al., Phys. Rev. Lett. 92, 226405 (2004); F. Dolcini et al., Phys. Rev. B 71, 165309 (2005); A. V. Lebedev, A. Crépieux, and Th. Martin, ibid. 71, 075416 (2005).

[5] R. Aguado and L. P. Kouwenhoven, Phys. Rev. Lett. 84, 1986 (2000); O. Astafiev et al., ibid. 93, 267007 (2004); J. P. Pekola et al., ibid. 95, 197004 (2005); R. J. Schoelkopf et al., in Ref. [2]; R. Deblock et al., Science 301, 203 (2003); J. Claudon et al. (unpublished).

[6] B. Reulet, J. Senzier, and D. E. Prober, Phys. Rev. Lett. 91, 196601 (2003); K. E. Nagaev, S. Pilgram, and M. Büttiker, ibid. 92, 176804 (2004); Yu. Bomze et al., ibid. 95, 176601 (2005); A. V. Galaktionov, D. S. Golubev, and A. D. Zaikin, Phys. Rev. B 68, 235333 (2003).

[7] M. Büttiker, Phys. Rev. B 46, 12485 (1992).

[8] We sum over repeated indices unless stated otherwise.

[9] V. A. Khlus, Zh. Eksp. Teor. Fiz. 93, 2179 (1987) [Sov. Phys. JETP 66, 1243 (1987)].

[10] B. Reulet and D. E. Prober, Phys. Rev. Lett. 95, 066602 (2005).

[11] M.L. Polianski and P. W. Brouwer, J. Phys. A 36, 3215 (2003).

[12] O. Agam, I. Aleiner, and A. Larkin, Phys. Rev. Lett. 85, 3153 (2000); F. Aigner, S. Rotter, and J. Burgdörfer, ibid. 94, 216801 (2005); M. Macucci et al., Phys. Rev. B 67, 115339 (2003).

[13] K. Flensberg, Phys. Rev. B 48, 11156 (1993); K. A. Matveev, ibid. 51, 1743 (1995); M. Kindermann and B. Trauzettel, Phys. Rev. Lett. 94, 166803 (2005).

[14] R. A. Jalabert, J.-L. Pichard, and C. W. J. Beenakker, Europhys. Lett. 27, 255 (1994); Ya. M. Blanter and E. V. Sukhorukov, Phys. Rev. Lett. 84, 1280 (2000).

[15] S. Oberholzer, E. V. Sukhorukov, and C. Schönenberger, Nature (London) 415, 765 (2002); S. Oberholzer et al., Phys. Rev. B 66, 233304 (2002).

[16] M. H. Pedersen, S. A. van Langen, and M. Büttiker, Phys. Rev. B 57, 1838 (1998).

[17] I. L. Kurland, I. L. Aleiner, and B.L. Altshuler, Phys. Rev. B 62, 14886 (2000); P. W. Brouwer, A. Lamacraft, and K. Flensberg, ibid. 72, 075316 (2005); Phys. Rev. Lett. 94, 136801 (2005).

[18] M. Büttiker, A. Prêtre, and H. Thomas, Phys. Rev. Lett. 70, 4114 (1993); A. Prêtre, H. Thomas, and M. Büttiker, Phys. Rev. B 54, 8130 (1996).

[19] M. Büttiker, J. Phys. Condens. Matter 5, 9361 (1993).

[20] P. W. Brouwer and M. Büttiker, Europhys. Lett. 37, 441 (1997).

[21] L. S. Levitov, H. W. Lee, and G. B. Lesovik, J. Math. Phys. (N.Y.) 37, 4845 (1996).

[22] K. E. Nagaev, Phys. Rev. B 57, 4628 (1998); Y. Naveh, D. V. Averin, and K. K Likharev, ibid. 59, 2848 (1999). 